\title{
Using sedatives-hypnotics or opioids in older adults: a narrow path
}

This issue of International Psychogeriatrics contains three papers from three continents that explore the use of sedatives-hypnotics and opioids in the treatment of older adults (Hamina et al., 2018; Machado-Duque et al., 2018; Tseng et al., 2018), and a fourth paper reporting on a qualitative study that addresses the broader question of training needs in dementia in a setting of rapid economic and demographic changes (Xu et al., 2018).

Tseng et al. (2018) report on a study that examined at a single time-point a community based sample of 3,978 participants aged 65 years and over and living in Taiwan. Use of sedative and hypnotic medications (sleep-promoting benzodiazepines, zolpidem, zopiclone, Western and Chinese herbal medicines, and over the counter drugs) was assessed through a self-report with confirmation where possible from prescription records. The prevalence within the last month was $19.7 \%$. The strongest binary predictor of use of any of these agents was the presence of any mental illness, followed by sleep problems, higher education levels, unemployment, depression, female gender, pain, being married, and current alcohol consumption. Continuous variables associated with use included the number of cardiovascular co-morbidities, number of non-cardiovascular co-morbidities, and age. Use of sedative-hypnotic medications was not associated with having cognitive problems: users had slightly but not significantly higher Folstein Mini-Mental State scores than non-users. The lack of a longitudinal component to the study means that risks of drug exposure cannot be evaluated prospectively. However, the absence of an association with cognitive problems is in line with the conclusions drawn by Gray et al. (2016). This similarly sized study allowed the impact of exposure to benzodiazepines, zolpidem, or in a small number of cases eszopiclone or zaleplon, to be evaluated over seven years of follow up; there was no consistent association between the use of sedative-hypnotic medication and subsequent development of Alzheimer's dementia. However, when specific aspects of cognitive performance were assessed in a recent meta-analysis (Crowe and Stranks, 2017), there was evidence of certain cognitive deficits being associated with chronic benzodiazepine use.

Sedatives and hypnotics are known to be associated with other risks in older adults, such as motor vehicle accidents and increased propensity to falls. Also in this issue, Machado-Duque et al. (2018) report on a case-control study of 861 older adults in Colombia, and describe an association between the use of benzodiazepines in the past month and falls with hip fractures, with an odds ratio (after adjustment for demographic factors and for other medications) of 3.73 (95\% CI $=1.60-$ 8.70). Still, the adjusted odds ratio for opioid medications was even higher, at 4.49 (95\% CI = 2.72-7.42).

In general, prescribers are advised to avoid exposing older adults to benzodiazepines and $\mathrm{z}-$ drugs (i.e. zaleplon, zolpidem, zopiclone, and eszopiclone) and to favor non-pharmacological approaches to treating insomnia, or else use alternative drugs (e.g. melatonin receptor agonists). However, as Tseng et al. (2018) and some guidelines (Baldwin et al., 2013) acknowledge, some individuals will experience good therapeutic effects from benzodiazepines or z-drugs, and for them the risk-benefit ratio of these drugs will be favorable, at least for a limited time. Some questions that have yet to be addressed are whether there is a specific dose or exposure threshold for benzodiazepines and $\mathrm{z}$-drugs below which risks may not be increased, or whether intermittent use over an extended period is associated with less adverse outcomes than chronic daily use.

Hamina et al. (2018) examined the impact of initiating opioid drugs on antipsychotic and benzodiazepine prescribing in people with Alzheimer's disease who live in the community. This is a population-based cohort study of 6,652 people, half of whom had started opioid medications, derived from data registers in Finland. Compared to those who did not initiate opioids, initiation of opioids was associated with an increase in the use of antipsychotics and benzodiazepines; however, after six months, there were small but significant reductions in use of both benzodiazepine and antipsychotic drugs in people who had initiated opioids. 
The rationale for this study is that good management of pain is essential in the care of people with Alzheimer's dementia. It is likely that some individuals with dementia who present with symptoms such as agitation and aggression are experiencing pain but cannot communicate this problem directly. In this situation, it is reasonable to expect that using an opioid to specifically achieve pain control is a better strategy than generically treating the indirect consequences of the pain (e.g. agitation and aggression). Thus, in this situation, opioids and other pain medications should be favored over drugs such as antipsychotics, which are otherwise the first line treatment for the behavioral and psychological symptoms of dementia (BPSD) (Davies et al., 2018). For example, in a randomized, double-blind, placebocontrolled, crossover trial in patients with moderate or severe dementia who presented with BPSD, the non-opioid analgesic agent acetaminophen (paracetamol) increased socialization but did not improve agitation relative to placebo (Chibnall et al., 2005). Unlike in Hamina et al.'s study, a concomitant reduction in the use of other medications was not observed. Perhaps, the explanation for this divergence is that opioids are more powerful analgesic medications than acetaminophen. Thus, they are more likely to reduce agitation and impact concomitant drug use when their introduction is targeted specifically for pain, as may have been the case in many patients in the community-based cohort of Hamina et al.'s study. If this is confirmed, the judicious use of opioids may improve the control of pain and allow the avoidance or discontinuation of other drugs otherwise prescribed for BPSD (e.g. antipsychotics or benzodiazepines). In contrast, if opioids or other analgesic medications are prescribed irrespective of the suspicion of pain, as was the case in Chibnall et al.'s study, reductions in agitation and in the use of antipsychotics or other medications targeting BPSD would be limited to those in whom agitation was a consequence of uncontrolled pain.

While opioid use may be beneficial in some circumstances, prescribers must be vigilant for undesirable effects, such as the associated increased risk of falls reported in this issue by MachadoDuque et al. (2018), and the potential for tolerance and dependence. Furthermore, an increase in the use of opioids in individuals with dementia would increase the likelihood that opioids are coprescribed with antipsychotics or benzodiazepines, exposing these frail patients to dangerous pharmacokinetic and pharmcodynamic interactions which can occur these medications are co-prescribed (FDA Drug Safety Communication, 2016). Thus, while we agree that opioids should be considered when agitation or aggression are strongly suspected to have arisen due to untreated pain, we do not believe that opioids should be used as a first line treatment in all cases of BPSD.

Finally, a paper by $\mathrm{Xu}$ et al. (2018) reports on the broader issue of how dementia care might be improved in China. As a rapidly developing nation, China is in the midst of demographic changes that will see the proportion of the population aged 65 years and over increase from $10.1 \%$ in 2015 to a projected $17.2 \%$ in 2030, amounting to 238 million individuals ( $\mathrm{He}$ et al., 2016). It is therefore imperative that as the number of individuals at risk of dementia increases, healthcare planners understand how resources can be targeted to allow those who will care for people with dementia to maximize the benefits they can deliver. Using a qualitative approach, the authors conducted focus group discussions with representatives from 40 Beijing mental health providers, differentiating the needs of "formal" or professional carers and those of "informal" carers such as family, friends, and neighbors. Themes relating to professional carers included the need for clinical knowledge about dementia and practical skills in its management, including skills relating to counseling and communication for caregivers. Meanwhile for the informal carers, themes relating to more basic necessities such as a rudimentary understanding of dementia and strategies for safety, maintenance of functioning, stress management, and communication skill were identified. Suggested strategies to implement change in professional practice, at both governmental and community levels, are discussed.

\section{Acknowledgments}

Within the past five years, Dr. Davies has received grant support from the Centre for Addiction and Mental Health (CAMH)/University of Toronto, NIHR (UK), Canadian Centre for Ageing and Brain Health Innovation, Canadian Consortium for Neurodegeneration in Aging (CCNA), and Medical-Psychiatry Alliance. Within the past five years, Dr. Mulsant has received research support from Brain Canada, the CAMH Foundation, the Canadian Institutes of Health Research, the National Institute of Health (NIH), PatientCentered Outcomes Research Institute (PCORI), Bristol-Myers Squibb (medication and matching placebo for a clinical trial funded by the US National Institute of Health - NIH), Eli Lilly (medication for an NIH-funded clinical trial), and Pfizer (medications and matching placebo for NIHfunded clinical trials). 


\section{Conflict of interest}

None.

Simon J. C. Davies ${ }^{1,2}$ and Benoit H. Mulsant ${ }^{1,2}$

${ }^{1}$ Centre for Addiction and Mental Health, Toronto, Ontario, Canada

${ }^{2}$ Department of Psychiatry, University of Toronto, Toronto, Ontario, Canada

Email: simon.davies@camh.ca;

benoit.mulsant@utoronto.ca

\section{References}

Baldwin, D. S. et al. (2013). Benzodiazepines: risks and benefits. A reconsideration. Fournal of Psychopharmacology, 27, 967-971.

Chibnall, J. T., Tait, R. C. and Harman, B. (2005). Effect of acetaminophen on behavior, well-being, and psychotropic medication use in nursing home residents with moderate-to-severe dementia. Fournal of the American Geriatrics Society, 53, 1921-1929.

Crowe, S. F. and Stranks, E. K. (2017). The residual medium and long-term cognitive effects of benzodiazepine use: an updated meta-analysis. Archives of Clinical Neuropsychology. Epublished ahead of print, doi:10.1093/arclin/acx120.

Davies, S. J. et al. (2018). Sequential drug treatment algorithm for agitation and aggression in Alzheimer's and mixed dementia. Fournal of Psychopharmacology, 32, 509-523.

FDA Drug Safety Communication (2016). FDA Warns About Serious Risks and Death When Combining Opioid Pain or Cough Medicines with Benzodiazepines; Requires Its Strongest Warning. Available at: https://www.fda.gov/Drugs/ DrugSafety/ucm518473.htm; accessed on April 30, 2018.

Gray, S. L. et al. (2016) Benzodiazepine use and risk of incident dementia or cognitive decline: prospective population based study. BMF, 352, 190 .

Hamina, A. et al. (2018). Impact of opioid initiation on antipsychotic and benzodiazepine and related drug use among persons with Alzheimer's disease. International Psychogeriatrics, 30, 947-956.

He, W., Goodkind, D. and Kowal, P. (2016). An Aging World: 2015, International Population Reports, P95/16-1. Washington, DC: U.S. Government Publishing Office.

Machado-Duque, M. E., Castaño-Montoya, J. P., Medina-Morales, D. A., Castro-Rodríguez, A., González-Montoya, A. and Machado-Alba, J. E. (2018). Association between the use of benzodiazepines and opioidswith the risk of falls and hip fractures in older adults. International Psychogeriatrics, 30, 941-946.

Tseng, H. Y. et al. (2018). Sedative-hypnotic drug use among community-dwelling elderly in Taiwan. International Psychogeriatrics, 30, 957-965.

Xu, L., Hsiao, H. Y., Denq, W. and Chi, I. (2018). Training needs for dementia care in China from theperspectives of mental health providers: who, what, and how. International Psychogeriatrics, 30, 929-940. 